\title{
A New Hybrid Butterfly Optimization Algorithm for Green Vehicle Routing Problem
}

\author{
Dana Marsetiya Utama $\mathbb{D}^{1},{ }^{1}$ Dian Setiya Widodo $\left(\mathbb{D},{ }^{2}\right.$ Muhammad Faisal Ibrahim $\left(\mathbb{D},{ }^{3}\right.$ \\ and Shanty Kusuma Dewi $\mathbb{D}^{1}$ \\ ${ }^{1}$ University of Muhammadiyah Malang, Jl. Tlogomas No. 246, 65144 Malang, East Java, Indonesia \\ ${ }^{2}$ University of 17 Agustus 1945 Surabaya, Jl. Semolowaru, Sukolilo, Surabaya 60118, East Java, Indonesia \\ ${ }^{3}$ Universitas Internasional Semen Indonesia, Jl. Veteran, Sidokumpul, Gresik 61122, East Java, Indonesia \\ Correspondence should be addressed to Dana Marsetiya Utama; dana@umm.ac.id
}

Received 25 March 2020; Revised 31 October 2020; Accepted 7 December 2020; Published 22 December 2020

Academic Editor: Kun An

Copyright ( $\odot 2020$ Dana Marsetiya Utama et al. This is an open access article distributed under the Creative Commons Attribution License, which permits unrestricted use, distribution, and reproduction in any medium, provided the original work is properly cited.

In the industrial sector, transportation plays an essential role in distribution. This activity impacts climate change and global warming. One of the critical problems in distribution is the green vehicle routing problem (G-VRP). This study focuses on G-VRP for a single distribution center. The objective function is to minimize the distribution costs by considering fuel costs, carbon costs, and vehicle use costs. This research aims to develop the hybrid butterfly optimization algorithm (HBOA) to minimize the distribution costs on G-VRP. It was inspired by the butterfly optimization algorithm (BOA), which was by combining the tabu search (TS) algorithm and local search swap and flip strategies. BOA is a new metaheuristic algorithm that has been successfully applied in various engineering fields. Experiments were carried out to test the parameters of the proposed algorithm and vary the speed of vehicles. The proposed algorithm was also compared with several procedures of prior study. The experimental results proved that the HBOA could minimize the total distribution cost compared to other algorithms. Moreover, the computation time is also included in the analysis.

\section{Introduction}

In distribution, transportation plays an essential role in the industrial sector. It proposes to distribute products from warehouses to customers [1]. Transport and logistics are also significant in the economic development of the world [2]. However, these activities impact climate change and global warming [3, 4]. In China, $30 \%$ of the total carbon emissions are caused by the goods transportation sector [5]. Furthermore, in the United States, $28.5 \%$ of greenhouse gas is caused by the transportation of goods [6]. Global climate change prevention was declared at the Copenhagen global climate summit in 2009 [7]. Generally, vehicles use fossil fuels as the source of engine-driving energy. Therefore, air pollution is mostly caused by the transportation sector $[8,9]$. One of the efforts to solve this problem is determining the right route. The problem of minimizing carbon emissions and fuel energy on this vehicle route is classified as a green vehicle routing problem (G-VRP) $[10,11]$. This issue has attracted the attention of many researchers $[12,13]$. G-VRP is the development of the classic vehicle routing problem (VRP). The VRP aims to minimize the total cost $[14,15]$ and the distance of travel [16-18]. However, the G-VRP aims at reducing the environmental impacts, such as reducing fuel consumption and carbon emissions caused by the distribution process $[19,20]$. Therefore, several effective procedures have been developed to solve G-VRP. In recent years, there has been an increase in research interest in this problem.

The researchers classify G-VRP as NP-hard problem [21-23]. They argue that the search for solutions to these problems is difficult to find at the time of polynomials. Recently, one popular procedure is metaheuristics [24]. Several researchers investigate the issue of G-VRP to reduce fuel consumption and carbon emissions partially. In the fuel consumption problem, some metaheuristic algorithms have been used to solve this problem. Cooray and Rupasinghe [25] proposed genetic algorithms (GA) to reduce energy 
consumption. Particle swarm optimization (PSO) was proposed by Poonthalir and Nadarajan [26] in a fuel-efficient G-VRP with varying speed. PSO was also used by Norouzi et al. [27] to minimize fuel consumption with time dependency. Zhang et al. [28] offered the ant colony optimization (ACO) algorithm for minimizing fuel consumption in multidepot. Other studies to minimize fuel consumption such as simulated annealing (SA) have been proposed by Kuo [29] and Normasari et al. [30], the revised hybrid intelligent algorithm was developed by Wang et al. [31], and Andelmin and Bartolini [32] offered the heuristic multistart local search procedure. Meanwhile, Macrina et al. [33] proposed a hybrid extensive neighborhood search, and Wang and Lu [10] developed the memetic algorithm with competition.

Besides, some researchers have resolved G-VRP to minimize carbon emissions. Some popular algorithms for this problem are GA [34, 35], tabu search (TS) [36-38], the Clarke and Wright algorithm [39], and GA with dynamic programming [40]. The differential evolution algorithm was developed by Kunnapapdeelert and Klinsrisuk [41] to solve G-VRP with pickup and delivery problems. Molina et al. [42] proposed the TS with neighborhood variables to reduce pollutant emissions. On the other hand, several studies on G-VRP to minimize carbon emissions and energy consumption simultaneously have been carried out successfully by researchers. Li et al. [43] proposed a modified PSO to reduce the total costs, including quality loss cost, vehicle operating cost, penalty cost, product freshness cost, emissions cost, and energy cost. TS was offered by Poonthalir and Nadarajan [26] to solve G-VRP by considering heterogeneous fixed fleet. Zhang et al. [7] also proposed the TS algorithm to reduce the total distribution costs, including fuel costs, carbon costs, and vehicle use costs. Hybrid GA was offered by Wang et al. [44] to minimize total cost distribution, which includes carbon emission costs. Shen et al. [45] developed PSO and TS to reduce minimum distribution costs, including penalty costs, the driver salary, fuel costs, and carbon emissions costs. Improved ACO algorithm was proposed by Li et al. [46], and Karagul et al. [47] employed the SA algorithm. Based on the trend of problem-solving methods, advanced metaheuristic algorithms have gained popularity in solving G-VRPs. Even a hybrid algorithm is used to solve this problem, and it has the advantage of solving NP-hard problems. Unfortunately, little research has addressed the use of a hybrid algorithm to solve G-VRP.

Recently, one of the advanced algorithms is the butterfly optimization algorithm (BOA). It is a new algorithm that can solve optimization problems proposed by Arora and Singh [48]. BOA has been effectively used to solve problems in various fields. Wen and Cao [49] applied a predicting model for exploring household $\mathrm{CO}_{2}$ emission mitigation strategies. BOA was implemented by Sharma et al. [50] in compression string design, welded beam design, and pressure vessel design. Yildiz et al. [51] used BOA to design automobile suspension components. Several studies have applied the BOA to solve several problems. However, there has not been any research about solving G-VRP using the hybrid butterfly optimization algorithm (HBOA). Those reasons motivate the author to conduct this research. Moreover, although some researchers have investigated G-VRP, minimizing carbon emissions and energy still receives little attention in the research literature. One interesting issue of G-VRP was investigated by Zhang et al. [7]. They solved the G-VRP to minimize the total distribution costs by considering fuel costs, carbon costs, and vehicle use costs with the TS algorithm. Unfortunately, the study of Zhang et al. [7] and previous studies do not consider computation time, and it is an essential aspect of optimization. Therefore, we propose the HBOA to minimize distribution costs that include fuel consumption, carbon emission, and vehicle use costs. There are two main objectives of this research: (1) developing the HBOA to minimize distribution costs of G-VRP and (2) comparing the performance of the proposed algorithm computation time. The HBOA was tested with several experiments to find out the best parameters. It is also compared to several algorithms. This research provides a significant contribution as the HBOA is a new algorithm in the G-VRP.

This paper structure is presented as follows: Section 2.1 describes assumptions, notations, and problem description; Section 2.2 explains the HBOA algorithm; Section 2.3 presents data and experimental procedures; Section 3 provides results and discussion; and Section 4 presents conclusions and future work.

\section{Materials and Methods}

2.1. Assumptions, Notations, and Problem Description. In this section, assumptions, notations, and problem descriptions are based on studies from Zhang et al. [7]. We consider transportation with one distribution center and a set of nodes. Vehicles have equal capacity (homogeneous). The distribution cost considered is fuel consumption cost, carbon emission cost, and a used vehicle cost. Highly total distribution costs require proper distribution management. Therefore, distribution centers need to manage the right transportation routes to minimize the total distribution costs. In this problem, vehicle fuel consumption is based on the distance traveled from node $s$ to node $s+1$. We assume that the weight of the additional load $M$ of the vehicle increases fuel consumption $p$ percent. Furthermore, the fuel consumption of unloaded vehicles is also considered in the total distribution costs. Assumptions, notations, and problem descriptions are described in the following section.

2.1.1. Assumptions and Notations. This study employed several assumptions in G-VRP, including the following: (1) the route begins and ends at the distribution center; (2) the costs consist of fuel consumption cost, carbon emission cost, and vehicle rental cost; (3) the vehicle has a fixed load capacity for each trip; (4) fuel, emissions, and vehicle usage costs are fixed; (5) vehicle speed is fixed; (6) the demand for each node is fixed; (7) each customer service time is fixed; and (8) this problem considers one distribution center. This study used notations to make it more practical to decipher the problem description. The notations are as follows: 
TDC: total distribution cost

$R_{r}^{s}$ : the $s$ th node on the $r$ th route (for example, $R_{2}^{3}=1$, the 2 path is $0-3-1-7-0$, and node in the 3 is 1 )

$d_{\left(R_{r}^{s}\right)\left(R_{r}^{s+1}\right)}$ : distance in $r$ th route from node $s$ to node $s+1$

$\mathrm{FC}_{\left(R_{r}^{s}\right)\left(R_{r}^{s+1}\right)}$ : total fuel consumption in $r$ th route from node $s$ to node $s+1$

$\mathrm{KPL}_{\left(R_{r}^{s}\right)\left(R_{r}^{s+1}\right)}$ : the traveled distance per unit fuel in $r$ th route from node $s$ to node $s+1$

$\mathrm{LPH}_{\left(R_{r}^{s}\right)\left(R_{r}^{s+1}\right)}$ : the fuel consumption per unit time of unload vehicle in $r$ th route from node $s$ to node $s+1$

$v_{\left(R_{r}^{s}\right)\left(R_{r}^{s+1}\right)}$ : the average speed of unloaded vehicles

$L_{\left(R_{r}^{s}\right)\left(R_{r}^{s+1}\right)}$ l load of vehicle in $r$ th route from $s$ th node to $s+1$ node

$M$ : additional load weight

$p$ : percentage increase of fuel

$N$ : number of vehicles or number of routes

$V_{r}$ : number of nodes on route $r, r=1,2, \ldots, N$

$s t_{R_{r}^{s}}$ : service time at the $s$ th node on the $r$ th route

$q_{R_{r}^{s}}$ : demand at the $s$ th node on the $r$ th route

$Q$ : vehicle capacity

$C_{f}$ : fuel consumption cost (fuel prices)

$C_{e}$ : emission carbon cost per unit of fuel consumption

$C_{V}$ : vehicle usage cost per unit time

2.1.2. Problem Description. This study made a mathematical model to describe the problem. The mathematical model is used to minimize distribution costs. The distribution costs considered are fuel cost, carbon emissions cost, and vehicle usage cost. Furthermore, the problem description illustrated is modeled as follows:

$$
\begin{aligned}
\operatorname{Min} \mathrm{TDC}= & \sum_{r=1}^{N} \sum_{s=1}^{v_{r}-1}\left(C_{f}+C_{e}\right) \times\left(\mathrm{LPH}_{\left(R_{r}^{s}\right)\left(R_{r}^{s+1}\right)}\right. \\
& \times \frac{d_{\left(R_{r}^{s}\right)\left(R_{r}^{s+1}\right)}}{V_{\left(R_{r}^{s}\right)\left(R_{r}^{s+1}\right)}} \times\left(1+p \times \frac{\left.\left.L_{\left(R_{r}^{s}\right)\left(R_{r}^{s+1}\right)}\right)\right)}{M}\right. \\
& +\sum_{r=1}^{N} \sum_{s=1}^{V_{r}-1} C_{V} \times\left(\frac{d_{\left(R_{r}^{s}\right)\left(R_{r}^{s+1}\right)}}{V_{\left(R_{r}^{s}\right)\left(R_{r}^{s+1}\right)}}+s t_{R_{r}^{s}}\right),
\end{aligned}
$$

subject to

$$
\begin{gathered}
\sum_{s=2}^{V_{r}-1} q_{R_{r}^{s}} \leq Q, \quad \forall r=1,2, \ldots, N, \\
L_{\left(R_{r}^{s}\right)\left(R_{r}^{s+1}\right)}=\sum_{s^{\prime}=s+1}^{V_{r}-1} q_{R_{r}^{\prime}}, \quad \forall r=1,2, \ldots, N, \\
R_{r}^{1}=R_{r}^{V_{r}}=0, \quad \forall r=1,2, \ldots, N,
\end{gathered}
$$

$N \geq 0, V_{r} \geq 0, R_{r}^{s} \subset V, \quad \forall r=1,2, \ldots, N, \forall s=1,2, \ldots, V_{r}$.

Equation (1) formulates the objective function in minimizing the total distribution cost, including vehicle use cost, fuel consumption cost, and carbon emissions cost. The cost of fuel consumption and carbon emissions cost considers the increase in fuel consumption $(p)$ for each additional load $(M)$. The fuel consumption per unit time of the unloading vehicle in $r$ th route from node $s$ to node $s+1$ also is considered. Constraints (2) and (3) describe formulas to ensure that the total load does not exceed the vehicle capacity. On each route $r$, the total vehicle load must not exceed the vehicle capacity. The total vehicle load must be ensured that it does not exceed the capacity. It becomes essential in the G-VRP. Constraint (4) shows that the first and last nodes of each vehicle route are the distribution center. As a G-VRP with one distribution center, this constraint ensures that each route starts at the distribution center and also ends at the distribution center. Constraint (5) formulates well-defined decision variables. This constraint defines the number of nodes and routes $\geq 0$, and it describes the decision variable at the sth node on the $r$ th route.

2.2. The Proposed Hybrid Butterfly Optimization Algorithm (HBOA). This study offered HBOA to solve G-VRP. The proposed algorithm was inspired by a BOA metaheuristic algorithm by combining the TS heuristic algorithm and the local search strategy. The main inspiration for the proposed algorithm was from BOA. The BOA was initially proposed by Arora and Singh [48] in 2019. There are two main characteristics in BOA, namely, the fragrance and movement of butterflies. These characteristics distinguish BOA from other algorithms. The basic BOA is shown in Figure 1.

Although the BOA has been proposed, this algorithm has not yet been satisfactory as it can only solve continuous problems. Meanwhile, the proposed algorithm is expected to solve G-VRP that constitutes sophisticated and discrete characteristics. G-VRP is categorized as an NP-hard combinatorial problem that must be addressed by a discrete search space. Therefore, this study offered a new approach to solve G-VRP. This research proposed five main steps on HBOA, such as (1) convert search agent position to travel order with large rank value (LRV), (2) change the position of $10 \%$ search agent based on the TS algorithm, (3) fragrance update, (4) movement of butterflies, and (5) local search. This study proposed an LRV procedure for converting continuous values to discrete values. To improve the BOA performance, this study combined TS and local search algorithms. Swap and flip rules were suggested in the local search strategy. The proposed algorithm is shown in Figure 2 . The five stages of the proposed algorithm are described in the following section.

2.2.1. Convert Search Agent Position to Travel Order with Large Rank Value (LRV). In this section, initializing the search agent position was generated randomly according to 


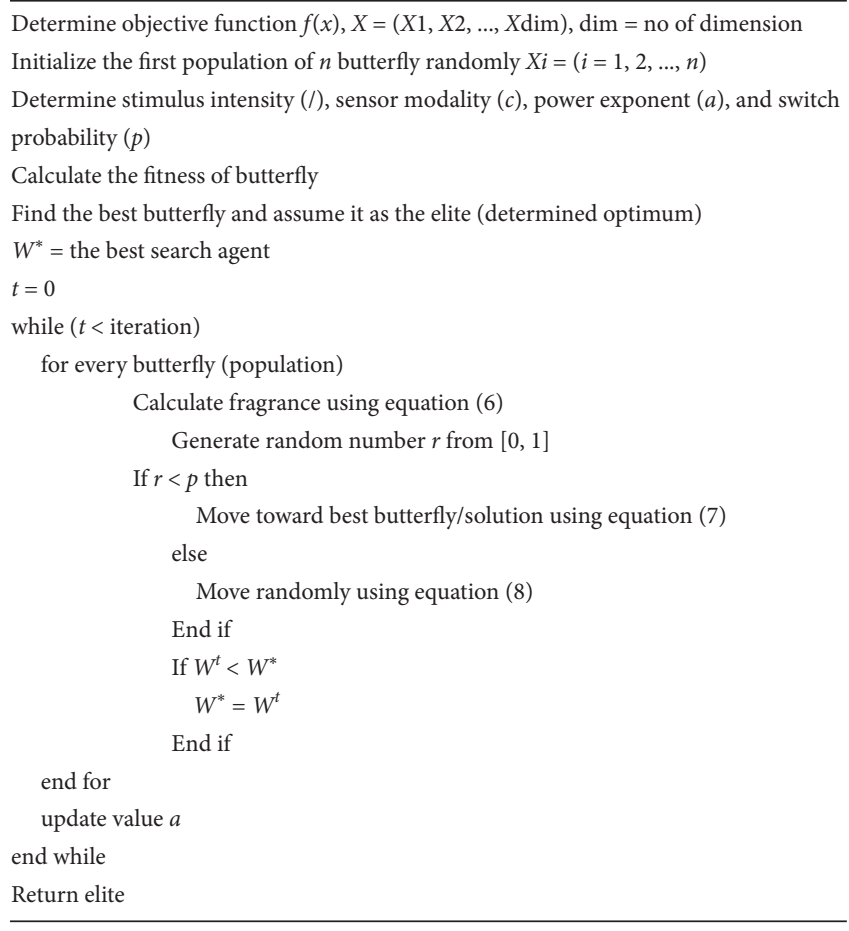

FIGURE 1: Pseudocode of butterfly optimization algorithm (BOA).

the upper and lower limits. The upper and lower limit values were set to determine the position of the BOA agent. At this stage, the search agent's position was ensured with no repeating numbers on the same search agent (Figure 3).

Furthermore, we proposed the principle of LRV to convert from the position of the search agent (continuous value) to the order of travel (discrete value). LRV is a popular method to convert from continuous value to discrete value in combinatorial problems [52-55]. At this stage, each search agent position value was sorted from the largest value to the smallest one. The LRV representation is shown in Figure 4. However, the illustration of Figure 4(b) could not be applied because, in one position, the search agent had the same value (0.43). In other words, the search agent position could not be applied as the order/route of vehicles visited the same two places.

2.2.2. Tabu Search Algorithm. In this section, this study proposed that $10 \%$ of the initial search agent positions were adjusted to the tabu search (TS) algorithm solution. The TS algorithm is a popular heuristic algorithm widely used to solve G-VRP. This study used the TS procedure developed by Poonthalir and Nadarajan [26]. To solve G-VRP, the five main stages [37] of the TS algorithm comprise (1) representation of solution, (2) initial solution, (3) neighborhood solution, (4) tabu list, and (5) criteria for aspiration and dismissal. Figure 5 represents the stages of the TS algorithm. The TS algorithm used three neighborhood solution rules. These rules were swap (Figure 6), flip (Figure 7), and slide (Figure 8). Swap is a rule in which it swaps two nodes. Flip is the rule of a node exchange by reversing the order of the node.

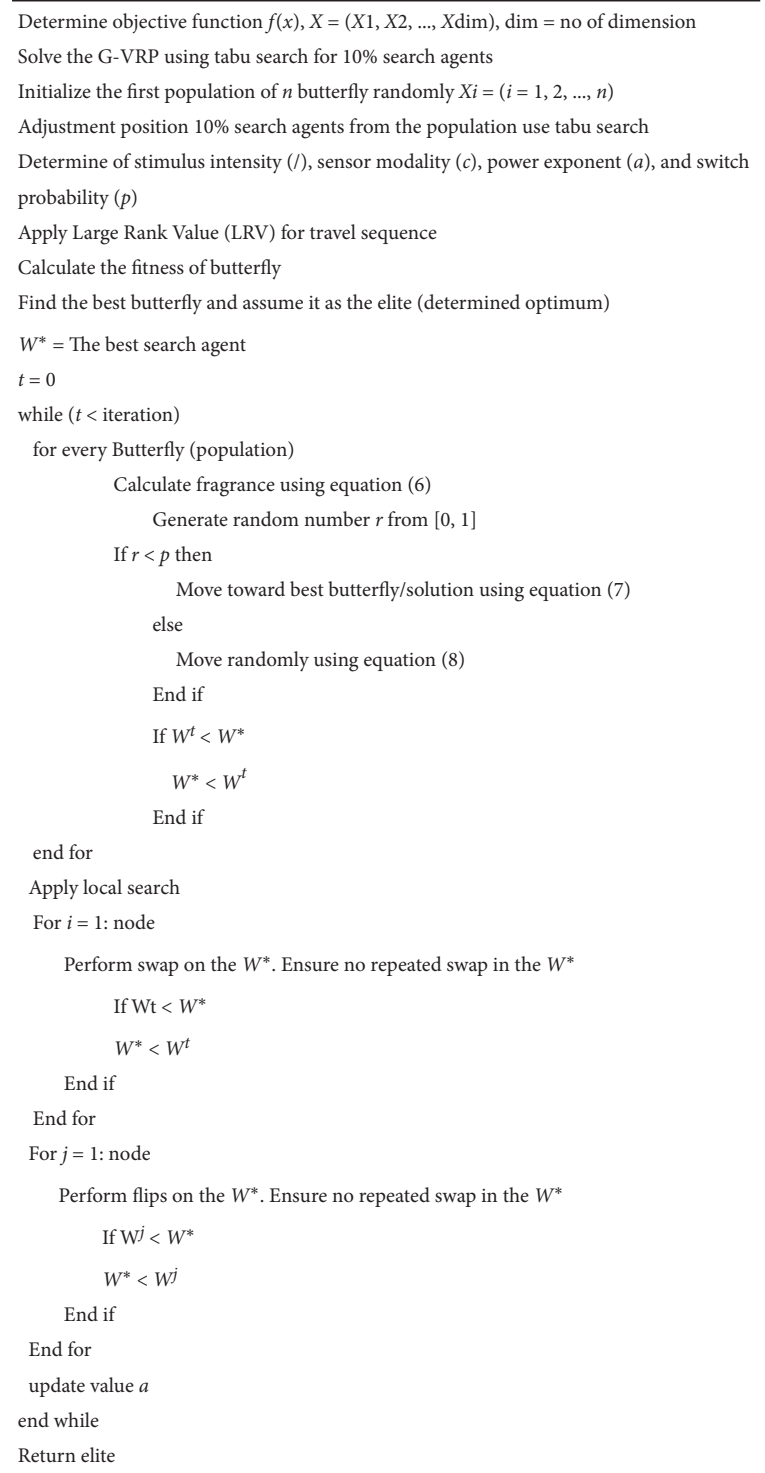

FIgURE 2: Pseudocode of hybrid butterfly optimization algorithm (HBOA).

Meanwhile, a slide is an exchange of nodes by shifting their sequences. For the $t$ th iteration to $t$, the swap and flip rules were repeated $n(n-1) / 2$ times. The slide rule was repeated $n^{2}$ times in each $t$ th iteration. For the solution inspection stage, the TS algorithm checked the tabu test by using the tabu list. It was to avoid repetition in finding a solution. In the aspiration criteria stage, the TS algorithm compared the new solution in the iteration $t$ to the previous solution in the iteration $t-1$. The new solution would be listed as the best solution if it had a better quality than that of the previous one. Furthermore, the stopping criteria used in the TS algorithm referred to the number of fulfilled iteration.

As mentioned earlier, this study proposed that $10 \%$ of search agent positions were adjusted to the TS algorithm's solution. Therefore, the position of the new search agent had to be adjusted to the TS solution. This study also suggested a new position adjustment procedure. The illustration of 


$$
\begin{array}{r}
\text { Populasi }=\left[\begin{array}{llll}
0,12 & 0,29 & 0,11 & 0,81 \\
0,61 & 0,43 & 0,88 & 0,76 \\
0,55 & 0,72 & 0,94 & 0,96
\end{array}\right] \\
\text { Populasi }=\left[\begin{array}{llll}
0,12 & \text { Accepted population } \\
0,61 & 0,33 & 0,71 & 0,71 \\
0,55 & 0,76 & 0,93 & 0,43 \\
0,73
\end{array}\right]
\end{array}
$$

(2) Rejected population

FIgURE 3: Initialization of the search agent position.

\begin{tabular}{|l|l|l|l|}
\hline 0.12 & 0.29 & 0.11 & 0.81 \\
\hline \multicolumn{2}{|l|}{} & \multicolumn{2}{|c|}{ Apply LRV } \\
\hline 3 & 2 & 4 & 1 \\
\hline
\end{tabular}

(a)

\begin{tabular}{|l|l|l|l|}
\hline 0.61 & 0.82 & 0.43 & 0.43 \\
\hline \multicolumn{2}{|l|}{} & \multicolumn{2}{|c|}{ Apply LRV } \\
\hline 2 & 1 & 3 & 3 \\
\hline
\end{tabular}

(b)

FIgURE 4: LRV representation. (a) Correct travel sequence and (b) wrong travel sequence.

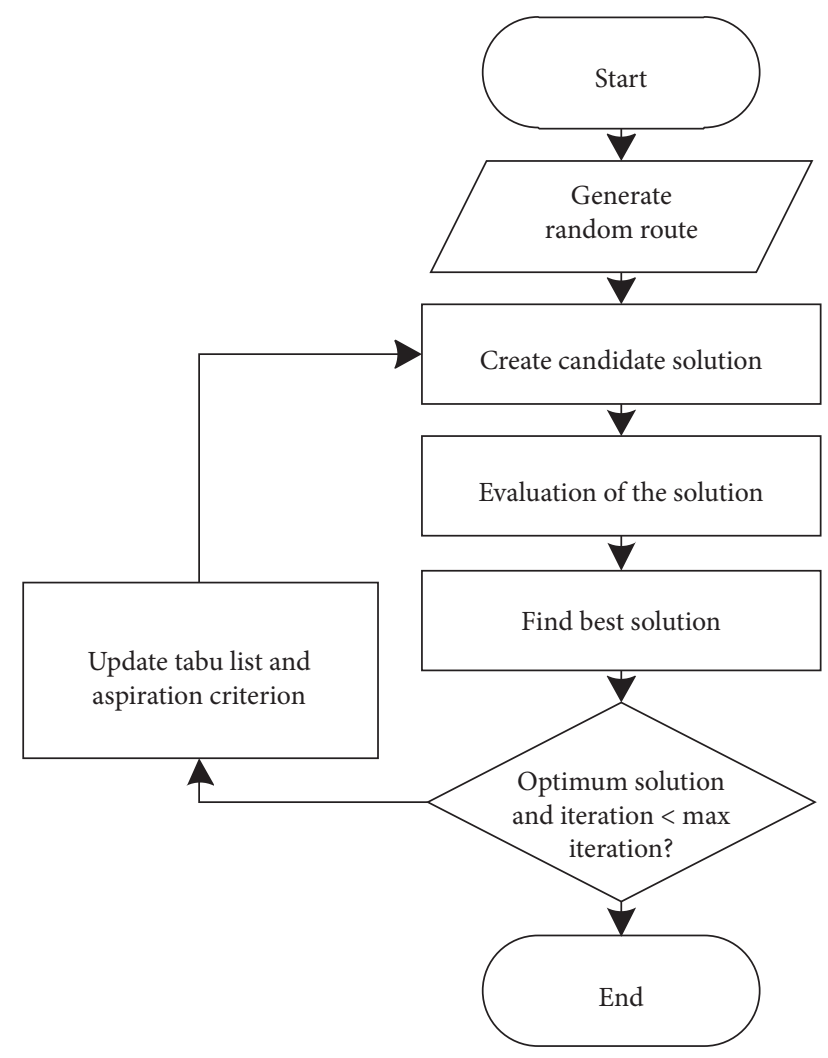

FIgURE 5: Flowchart of the tabu search algorithm.

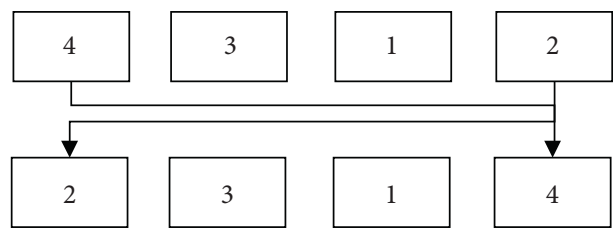

Figure 6: Swap illustration.

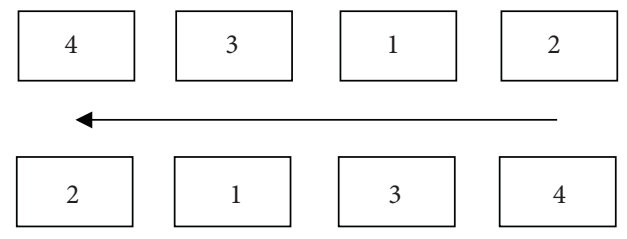

Figure 7: Flip illustration.

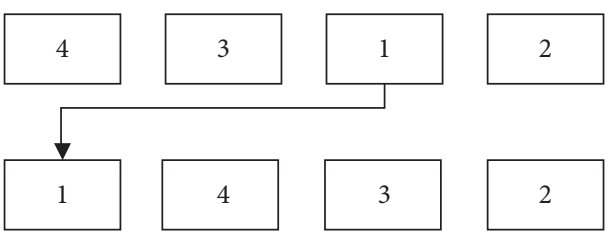

FIgURE 8: Slide illustration.

converting TS solutions to the position of the search agent is exemplified in Figure 9.

2.2.3. Update Fragrance. In BOA, each butterfly has a unique fragrance and personality. It is one of the main characteristics that distinguish BOA from any other metaheuristic algorithms. All BOA behaviors are based on the sensory modality $(c)$, stimulus intensity $(I)$, and exponential strength $(a)$. Fragrance $(f)$ is formulated as a function of the physical intensity of the stimulus from BOA. The $f$ formula is presented as follows:

$$
f=c \cdot I^{a}
$$

where $f$ is the value of fragrance that changes in every iteration. This value shows how strong the fragrance is felt by other butterflies. The butterfly stimulus intensity is formulated as $I$. $a$ is the power exponent that depends on the modality. $c$ formulates the sensory modality. Values of $a$ and $c$ on the used butterfly are in the range $[0,1]$. 


\begin{tabular}{|}
\begin{tabular}{|c|c|c|c|c|c|}
\hline $\begin{array}{c}\text { Route solution by tabu } \\
\text { search }\end{array}$ & 2 & 1 & 4 & 3 & 5 \\
\hline $\begin{array}{c}\text { Initial position search } \\
\text { agent }\end{array}$ & -8.0492 & -4.4300 & 0.9376 & 9.1051 & 9.2978 \\
\hline $\begin{array}{c}\text { New position search } \\
\text { agent position based on } \\
\text { tabu search }\end{array}$ & 9.1051 & $\downarrow$ & $\downarrow$ Position adjustment of search agent based on tabu search \\
\hline
\end{tabular} \\
\hline
\end{tabular}

FIGURE 9: Illustration of tabu search converted solution to the position of search agent.

2.2.4. Movement of Butterflies. This section explains the phase of the movement of butterflies. There are two main phases in the basic BOA, namely, the initial phase and the movement of the butterfly phase. In the butterfly phase movement, the butterflies move their position as many times as the number of iterations. In this phase, all butterflies in the solution room move to a new position. Then, the fitness value of each butterfly is evaluated. In each iteration, the fitness value of all butterflies is updated. Furthermore, the butterflies produce fragrance in their calculated position based on equation (6). Two movements in BOA are the global search phase and the local search phase. In the global search phase, butterflies take steps towards other butterflies that have the best solution. The global search phase for butterflies is represented in equation (7). Meanwhile, the local search phase is shown in equation (8):

$$
X_{i}^{t+1}=X_{i}^{t}+\left(r^{2} g^{*}-X_{i}^{t}\right) f_{i},
$$

where $X_{i}^{t}$ is the vector solution $X_{i}$ for the $i$ th butterfly in the iteration $t$. $r$ shows a random number in the range $[0,1] . g^{*}$ indicates the best solution in the current iteration. The $i$ th butterfly fragrance is represented by $f_{i}$.

$$
X_{k}^{t+1}=X_{i}^{t}+\left(r^{2} X_{j}^{t}-X_{k}^{t}\right) f_{i} .
$$

Equation (8) indicates the local butterfly search formula. $X_{j}^{t}$ and $X_{k}^{t}$ are the $j$-th and $k$-th butterflies from the solution room. $r$ is the random number in the range $[0,1]$. Movement of butterflies stops until the termination criteria are met. The stopping criterion used is the maximum number of the achieved iteration. After the movement of butterflies, the algorithm produces the best solution based on the fitness values.

2.2.5. Local Search. To improve the BOA performance, this study proposed the local search procedure. Swap and flip were the two local search rules chosen to improve the BOA performance. Figure 6 illustrates the proposed swap rules. In this rule, two positions (nodes) were chosen randomly and exchanged. Another local search rule used was flip. In this rule, two nodes were selected randomly and continued to reverse the order of the selected nodes. This rule is illustrated in Figure 7. In the proposed HBOA, for each iteration $t$, the swap and flip operations were repeated as many as the number of nodes.

\subsection{Data and Experimental Procedure}

2.3.1. Data. In this study, the data of the number of nodes, coordinates, vehicle capacity, and demand were taken from Gaskell [56] and Christofides and Eilon [57]. They used cases with nodes as many as 22 nodes (Table 1) [56], 32 nodes (Table 2) [57], and 50 nodes (Table 3) [57]. Distance $\left(d_{\left(R_{r}^{s}\right)\left(R_{r}^{s+1}\right)}\right)$ in $r$ th route from node $s$ to node $s+1$ is based on formula $d_{\left(R_{r}^{s}\right)\left(R_{r}^{s+1}\right)}=\sqrt{\left(X_{s}-X_{s+1}\right)^{2}+\left(Y_{s}-Y_{s+1}\right)^{2}}$. Meanwhile, the data of the costs and speed data were obtained from Zhang et al. [7]. The fuel cost was 7.3 yuan/liter [7]. The carbon emissions were 0.64 yuan/liter [7]. Furthermore, the vehicle usage fee was 80 yuan/hour [7]. This research employed three categories of vehicle speed (high, medium, and low speed). The high, medium, and low speeds were $107 \mathrm{~km} / \mathrm{hr}, 63 \mathrm{~km} / \mathrm{hr}$, and $43 \mathrm{~km} / \mathrm{hr}$, respectively. Nine variations of problems (three nodes and three-speed variations) were carried out in this study. Service time for each customer is 0.1 hours. The increase in fuel consumption $(p)$ for each additional load $M=50$ is $2 \%$.

2.3.2. Experimental Procedure. The experiments were designed to determine the effect of HBOA (iteration and population) and speed parameters on the distribution cost and computation time. The experiments were carried out with different parameters. The parameters included the number of populations and iterations. The population parameters used three different levels $(10,50$, and 100 populations). The iteration parameters also employed three levels (10,50, and 100 iterations). This study used the sensory modality of 0.01 and power exponent of 0.1 from BOA parameters. Eighty-one trials were designed in this study. Each result of the trial was recorded for cost and computation time.

The HBOA was compared to other algorithms such as BOA [48], TS [7], SA [29], ACO [28], PSO [27], and GA [58]. To compare with several algorithms, this study used one hundred iteration parameters at each vehicle speed in every algorithm. One hundred populations were used in the BOA experiment. Moreover, we used an initial temperature parameter of 1000 and the cooldown factor based on the Kuo [29] formula. One hundred ant populations were adopted for the ACO algorithm. One hundred particles and an inertia weight of 0.5 are used in the PSO algorithm. 100 populations, 
TABle 1: Problem of 22 nodes.

\begin{tabular}{|c|c|c|c|}
\hline \multirow{2}{*}{ Node } & \multicolumn{2}{|c|}{ Coordinate } & \multirow{2}{*}{ Demand } \\
\hline & $X$ & $Y$ & \\
\hline Depot & 266 & 235 & 0 \\
\hline 1 & 295 & 272 & 125 \\
\hline 2 & 301 & 258 & 84 \\
\hline 3 & 309 & 260 & 60 \\
\hline 4 & 217 & 274 & 500 \\
\hline 5 & 218 & 278 & 300 \\
\hline 6 & 282 & 267 & 175 \\
\hline 7 & 242 & 249 & 350 \\
\hline 8 & 230 & 262 & 150 \\
\hline 9 & 249 & 268 & 1100 \\
\hline 10 & 256 & 267 & 4100 \\
\hline 11 & 265 & 257 & 225 \\
\hline 12 & 267 & 242 & 300 \\
\hline 13 & 259 & 265 & 250 \\
\hline 14 & 315 & 233 & 500 \\
\hline 15 & 329 & 252 & 150 \\
\hline 16 & 318 & 252 & 100 \\
\hline 17 & 329 & 224 & 250 \\
\hline 18 & 267 & 213 & 120 \\
\hline 19 & 275 & 192 & 600 \\
\hline 20 & 303 & 201 & 500 \\
\hline 21 & 208 & 217 & 175 \\
\hline 22 & 326 & 181 & 75 \\
\hline
\end{tabular}

Capacity: 4500 .

a crossover probability of 0.8 , and a mutation probability of 0.25 were applied in the GA algorithm experiment. The performance was measured using relative error percentage (REP) as presented in equation (9). A positive REP showed that the proposed algorithm is better than the other algorithms. However, a negative REP showed that the proposed algorithm is not competitive as compared to other algorithms.

$$
\mathrm{REP}=\frac{\text { cost }_{\text {other algorithms }}-\text { cost }_{\text {proposed algorithm }}}{\text { cost }_{\text {proposed algorithm }}} \times 100 \% .
$$

Besides, this study also compared the computation time in all cases. It was carried out to determine the time efficiency of solving G-VRP. The effect of iteration $(t)$ on distribution costs was also analyzed. This analysis was carried out in 50 nodes, 100 populations, and 100 iterations in the case of medium-speed vehicles. Furthermore, all experiments were conducted with the means of Matlab R2014a software on Windows 8 Intel Celeron with x64-64 2GB RAM processor.

\section{Results and Discussion}

3.1. The Comparison of Varied Parameters and Speed towards Costs. Table 4 shows the results obtained from eighty-one experiments with variations of nodes, speed, iteration, and population. It shows that the minimum distribution cost solution is produced in the population parameters and high iterations. Therefore, overall, these results suggest that population parameters and significant iterations effectively minimize distribution costs for G-VRPs . It is interesting to note that with successive increases in both iteration and population, the distribution cost declined. It shows that the number of iterations and large population minimized the total costs. Besides, the speed of the vehicle affects the total distribution cost. Low speed requires high distribution costs. Average distribution costs are produced from mediumspeed vehicles. However, high speed results in small distribution costs. Therefore, this result shows that the high speed reduced the total costs in the case of G-VRP. Overall, these findings are consistent with the findings reported by Zhang et al. [7]. Overall, these findings are consistent with Zhang et al. [7], which indicate variation, speed, iteration, and population effect in the distribution cost.

The results of the iteration $(t)$ effect on the distribution costs are shown in Figure 10. It illustrates the algorithm effectiveness that can be seen from the impact of iteration on the distribution costs. From the data in Figure 10, the cost of distribution decreased as the iteration was added. Besides, the effect of iteration on the distribution costs shows that the convergence curve on $\mathrm{HBOA}$ is better than other algorithms. The HBOA produces better total distribution in each iteration compared to BOA [48], TS [7], SA [29], ACO [28], PSO [27], and GA [58]. The results of this study indicate that the proposed algorithm is effectively used to solve the G-VRP.

3.2. The Comparison of Varied Parameters and Speed towards Computation Time. Table 5 illustrates the experimental comparison between the varied parameters and speed towards the computation time. Small populations and iterations result in less computation time. However, large populations and iterations require considerable 
TABle 2: Problem of 32 nodes.

\begin{tabular}{|c|c|c|c|}
\hline \multirow{2}{*}{ Node } & \multicolumn{2}{|c|}{ Coordinate } & \multirow{2}{*}{ Demand } \\
\hline & $X$ & $Y$ & \\
\hline Depot & 292 & 425 & 0 \\
\hline 1 & 298 & 427 & 700 \\
\hline 2 & 309 & 445 & 400 \\
\hline 3 & 307 & 464 & 400 \\
\hline 4 & 336 & 475 & 1200 \\
\hline 5 & 320 & 439 & 40 \\
\hline 6 & 321 & 437 & 80 \\
\hline 7 & 322 & 437 & 2000 \\
\hline 8 & 323 & 433 & 900 \\
\hline 9 & 324 & 433 & 600 \\
\hline 10 & 323 & 429 & 750 \\
\hline 11 & 314 & 435 & 1500 \\
\hline 12 & 311 & 442 & 150 \\
\hline 13 & 304 & 427 & 250 \\
\hline 14 & 293 & 421 & 1600 \\
\hline 15 & 296 & 418 & 450 \\
\hline 16 & 261 & 384 & 700 \\
\hline 17 & 297 & 410 & 550 \\
\hline 18 & 315 & 407 & 650 \\
\hline 19 & 314 & 406 & 200 \\
\hline 20 & 321 & 391 & 400 \\
\hline 21 & 321 & 398 & 300 \\
\hline 22 & 314 & 394 & 1300 \\
\hline 23 & 313 & 378 & 700 \\
\hline 24 & 304 & 382 & 750 \\
\hline 25 & 295 & 402 & 1400 \\
\hline 26 & 283 & 406 & 4000 \\
\hline 27 & 279 & 399 & 600 \\
\hline 28 & 271 & 401 & 1000 \\
\hline 29 & 264 & 414 & 500 \\
\hline 30 & 277 & 439 & 2500 \\
\hline 31 & 290 & 434 & 1700 \\
\hline 32 & 319 & 433 & 1100 \\
\hline
\end{tabular}

Capacity: 8000 .

computation time. Therefore, the most apparent finding that emerges from the analysis is that the number of iteration and large population increased the computational time. Another important finding is that different vehicle speed did not appear to affect the computation time. Low speed, medium speed, and high speed produce relatively the same computation time.

Furthermore, the number of nodes affected the computational time. Cases with 22 nodes need little computation time. However, in the case of 50 nodes, the computation time required is considerable. Therefore, the experimental results show that the number of nodes increased the computing time. The comparison between the proposed algorithm's computation time and several other algorithms in the medium speed is presented in Figure 11. It can be seen that the proposed algorithm provided a relatively higher computation time as compared to several other algorithms, such as BOA [48], TS [7], SA [29], ACO [28], PSO [27], and GA [58]. Besides, the addition of nodes also increased the time significantly. Therefore, it can be concluded that the number of nodes has a significant effect on the computational time. It confirms the findings of Oesterle and
Bauernhansl [21] and Braekers et al. [23], stating that VRP is an NP-hard problem. Based on these results, further research is expected to be carried out to reduce the computation time so that the algorithm may become more efficient.

Although HBOA produces considerable computation time, the resulting total distribution costs are minimal. The small total cost of distribution is one of the most critical decisions in operations management. Decision-makers prefer to choose decisions with minimal total distribution costs because they provide benefits. Conversely, decisionmakers pay less attention to computation time because short computing time does not guarantee a minimal total distribution cost.

3.3. The Comparison of Algorithms. Table 6 shows the comparison of the REP values between the proposed algorithm and other algorithms. As shown in Figure 3, the REP values (based on equation (9)) of BOA [48], TS [7], SA [29], ACO [28], PSO [27], and GA [58] were 9\%, 46\%, 27\%, $31 \%, 28 \%$, and $23 \%$, respectively. The positive values from $\mathrm{REP}$ indicate that the proposed algorithm is more effective in 
TABle 3: Problem of 50 nodes.

\begin{tabular}{|c|c|c|c|}
\hline \multirow{2}{*}{ Node } & \multicolumn{2}{|c|}{ Coordinate } & \multirow{2}{*}{ Demand } \\
\hline & $X$ & $Y$ & \\
\hline Depot & 30 & 40 & 0 \\
\hline 1 & 37 & 52 & 7 \\
\hline 2 & 49 & 49 & 30 \\
\hline 3 & 52 & 64 & 16 \\
\hline 4 & 20 & 26 & 9 \\
\hline 5 & 40 & 30 & 21 \\
\hline 6 & 21 & 47 & 15 \\
\hline 7 & 17 & 63 & 19 \\
\hline 8 & 31 & 62 & 23 \\
\hline 9 & 52 & 33 & 11 \\
\hline 10 & 51 & 21 & 5 \\
\hline 11 & 42 & 41 & 19 \\
\hline 12 & 31 & 32 & 29 \\
\hline 13 & 5 & 25 & 23 \\
\hline 14 & 12 & 42 & 21 \\
\hline 15 & 36 & 16 & 10 \\
\hline 16 & 52 & 41 & 15 \\
\hline 17 & 27 & 23 & 3 \\
\hline 18 & 17 & 33 & 41 \\
\hline 19 & 13 & 13 & 9 \\
\hline 20 & 57 & 58 & 28 \\
\hline 21 & 62 & 42 & 8 \\
\hline 22 & 42 & 57 & 8 \\
\hline 23 & 16 & 57 & 16 \\
\hline 24 & 8 & 52 & 10 \\
\hline 25 & 7 & 38 & 28 \\
\hline 26 & 27 & 68 & 7 \\
\hline 27 & 30 & 48 & 15 \\
\hline 28 & 43 & 67 & 14 \\
\hline 29 & 58 & 48 & 6 \\
\hline 30 & 58 & 27 & 19 \\
\hline 31 & 37 & 69 & 11 \\
\hline 32 & 38 & 46 & 12 \\
\hline 33 & 46 & 10 & 23 \\
\hline 34 & 61 & 33 & 26 \\
\hline 35 & 62 & 63 & 17 \\
\hline 36 & 63 & 69 & 6 \\
\hline 37 & 32 & 22 & 9 \\
\hline 38 & 45 & 35 & 15 \\
\hline 39 & 59 & 15 & 14 \\
\hline 40 & 5 & 6 & 7 \\
\hline 41 & 10 & 17 & 27 \\
\hline 42 & 21 & 10 & 13 \\
\hline 43 & 5 & 64 & 11 \\
\hline 44 & 30 & 15 & 16 \\
\hline 45 & 39 & 10 & 10 \\
\hline 46 & 32 & 39 & 5 \\
\hline 47 & 25 & 32 & 25 \\
\hline 48 & 25 & 55 & 17 \\
\hline 49 & 48 & 28 & 18 \\
\hline 50 & 56 & 37 & 10 \\
\hline
\end{tabular}

Capacity: 80 .

solving G-VRP. Data processing results show that there is no REP average, which results in a negative REP value in the comparison algorithm. The order of the algorithm that has the smallest to largest positive REP is BOA [48], GA [58], SA [29], PSO [27], ACO [28], and TS [7]. Thus, the findings confirm that HBOA is more competitive as compared to other algorithms. In other words, HBOA can significantly improve the quality of the G-VRP solution.

The experimental results show that the HBOA can produce a minimal total distribution cost. This result is an 
TABLE 4: Results of the comparison between varied parameter and speed towards costs (yuan).

\begin{tabular}{|c|c|c|c|c|c|c|c|c|c|c|}
\hline \multirow{3}{*}{$V_{r}$} & \multirow{3}{*}{ Iteration } & \multirow{2}{*}{\multicolumn{3}{|c|}{$\begin{array}{l}\text { High speed } \\
\text { Population }\end{array}$}} & \multirow{2}{*}{\multicolumn{3}{|c|}{$\begin{array}{l}\text { Medium speed } \\
\text { Population }\end{array}$}} & \multirow{2}{*}{\multicolumn{3}{|c|}{$\begin{array}{l}\text { Low speed } \\
\text { Population }\end{array}$}} \\
\hline & & & & & & & & & & \\
\hline & & 10 & 50 & 100 & 10 & 50 & 100 & 10 & 50 & 100 \\
\hline \multirow{3}{*}{22} & 10 & 2814.4 & 2813.1 & 2385.9 & 2971.5 & 2967.7 & 2654 & 3949.6 & 3621.7 & 3442 \\
\hline & 50 & 2786.3 & 2666.9 & 2403.5 & 2909.3 & 2587.5 & 2518.5 & 3739.3 & 3577 & 3180.5 \\
\hline & 100 & 2211.8 & 2161.0 & 2020.7 & 2857.2 & 2423.6 & 2300.9 & 3511.1 & 3065.7 & 2808 \\
\hline \multirow{3}{*}{32} & 10 & 3392.6 & 2806.5 & 2687.4 & 3955 & 3777 & 3261.5 & 4487.9 & 3889.5 & 3753.8 \\
\hline & 50 & 2955.9 & 2732.6 & 2412.9 & 3627.3 & 3295.9 & 3068.5 & 4171.3 & 3657.5 & 3049.6 \\
\hline & 100 & 2922.1 & 2647.3 & 2399.6 & 3116.6 & 2836.3 & 2614.5 & 3442.3 & 2922.2 & 2998.3 \\
\hline \multirow{3}{*}{50} & 10 & 7629.0 & 7398.3 & 6950.1 & 9127 & 8528.5 & 8381.7 & 11391 & 11078 & 10095 \\
\hline & 50 & 7090.3 & 7002.6 & 6289.5 & 9090 & 8321.3 & 7987 & 10991 & 9806 & 9066 \\
\hline & 100 & 6876.9 & 6614.3 & 6097.2 & 8883 & 8193 & 7327.4 & 10423 & 9333 & 8836 \\
\hline
\end{tabular}

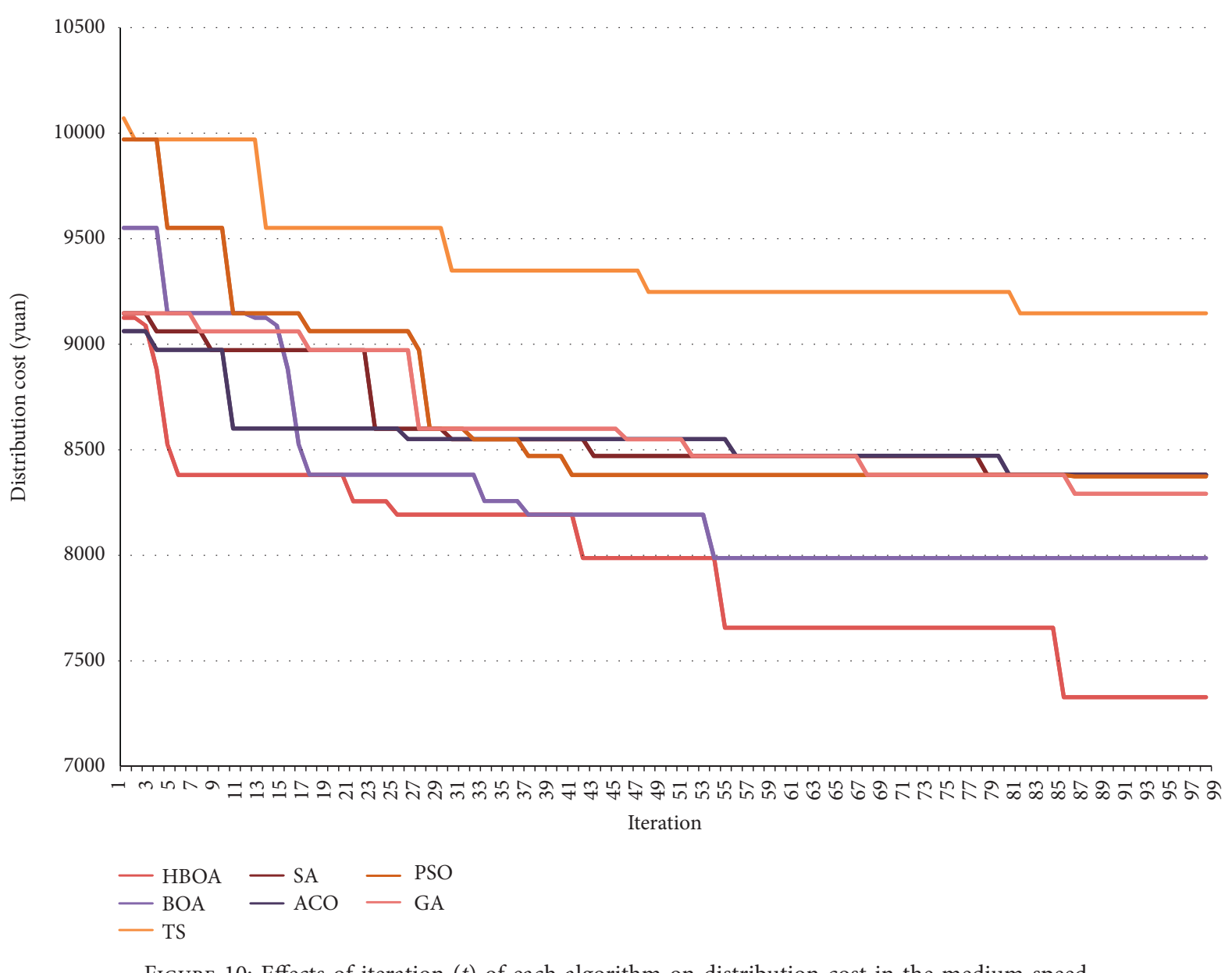

FIGURE 10: Effects of iteration $(t)$ of each algorithm on distribution cost in the medium speed. 
TABLE 5: Results of the comparison between varied parameters and speed towards computation time (seconds).

\begin{tabular}{|c|c|c|c|c|c|c|c|c|c|c|}
\hline \multirow{3}{*}{$V_{r}$} & \multirow{3}{*}{ Iteration } & \multirow{2}{*}{\multicolumn{3}{|c|}{$\begin{array}{l}\text { High speed } \\
\text { Population }\end{array}$}} & \multirow{2}{*}{\multicolumn{3}{|c|}{$\begin{array}{l}\text { Medium speed } \\
\text { Population }\end{array}$}} & \multirow{2}{*}{\multicolumn{3}{|c|}{$\begin{array}{l}\text { Low speed } \\
\text { Population }\end{array}$}} \\
\hline & & & & & & & & & & \\
\hline & & 10 & 50 & 100 & 10 & 50 & 100 & 10 & 50 & 100 \\
\hline \multirow{3}{*}{22} & 10 & 21.68 & 110.05 & 305.19 & 21.76 & 110.17 & 305.51 & 21.76 & 110.41 & 305.96 \\
\hline & 50 & 111.79 & 512.59 & 707.73 & 113.37 & 513.59 & 707.91 & 114.71 & 515.14 & 709.35 \\
\hline & 100 & 205.34 & 1139.50 & 1334.64 & 206.36 & 1140.88 & 1336.20 & 206.45 & 1141.16 & 1337.93 \\
\hline \multirow{3}{*}{32} & 10 & 26.98 & 116.47 & 312.67 & 27.05 & 116.58 & 312.98 & 27.06 & 116.83 & 313.44 \\
\hline & 50 & 143.18 & 546.20 & 747.43 & 144.76 & 547.20 & 747.61 & 146.09 & 548.74 & 749.05 \\
\hline & 100 & 267.98 & 1212.40 & 1413.01 & 269.00 & 1213.78 & 1414.57 & 269.09 & 1214.06 & 1416.30 \\
\hline \multirow{3}{*}{50} & 10 & 32.28 & 122.88 & 320.14 & 32.35 & 123.00 & 320.46 & 32.36 & 123.25 & 320.91 \\
\hline & 50 & 174.57 & 579.81 & 787.13 & 176.15 & 580.81 & 787.31 & 177.48 & 582.35 & 788.75 \\
\hline & 100 & 330.62 & 1285.30 & 1491.37 & 331.64 & 1286.68 & 1492.93 & 331.73 & 1286.96 & 1494.66 \\
\hline
\end{tabular}

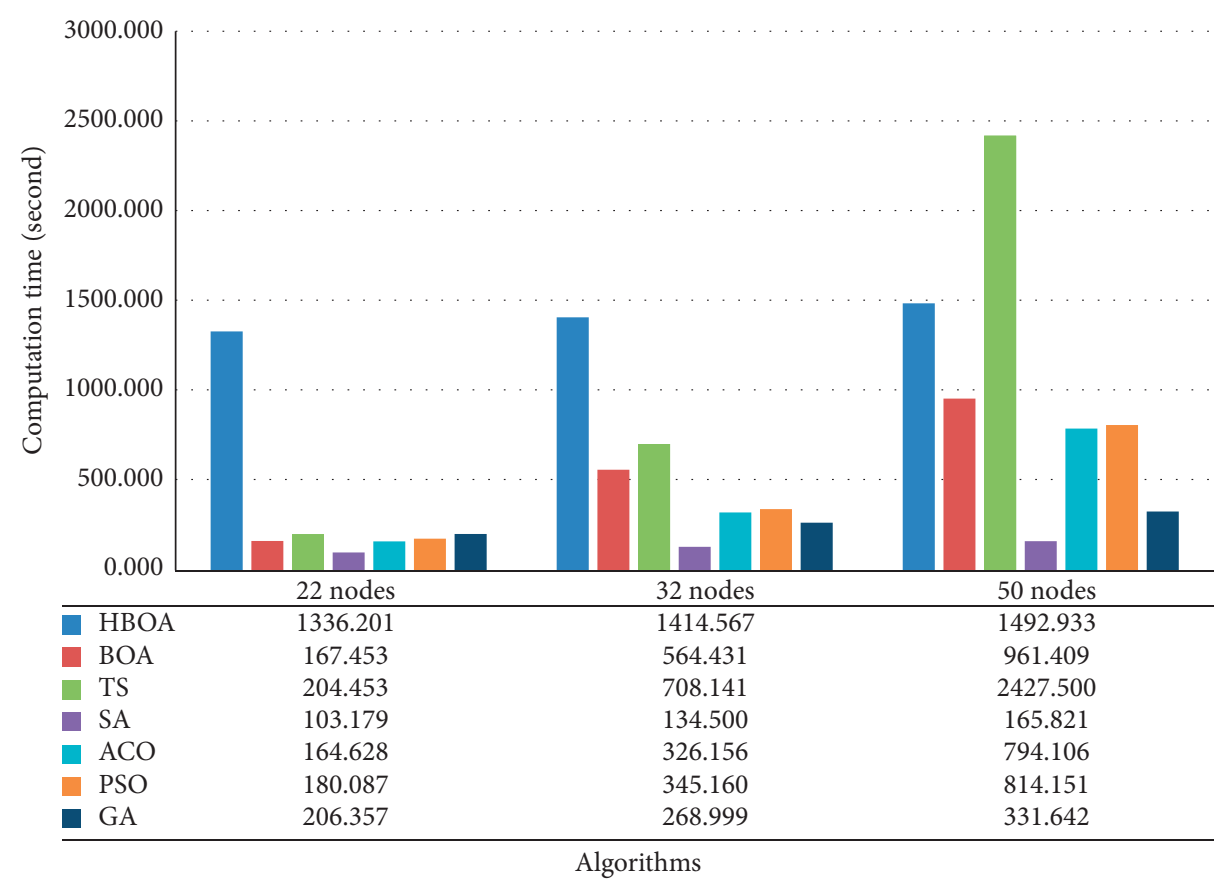

FIGURE 11: Comparison between the computation time of the proposed algorithm and several other algorithms in the medium speed.

TABLE 6: Comparison of distribution cost and the relative error percentage (REP) values between the proposed algorithm and other algorithms.

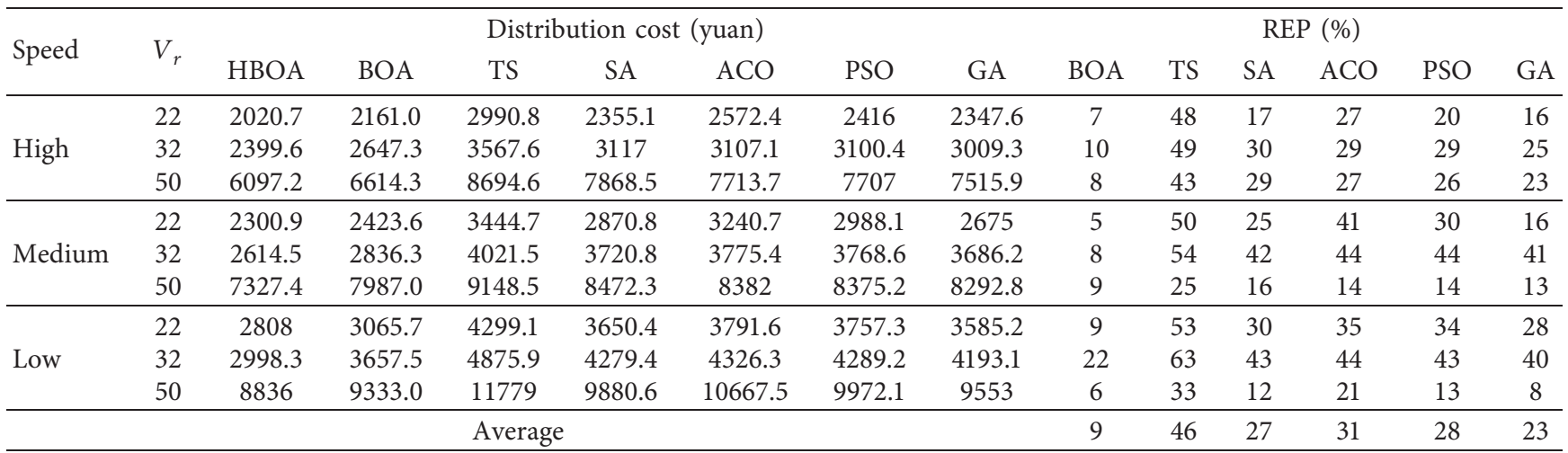


essential strength of the HBO algorithm. Unfortunately, there is a contradiction in the resulting computation time. The $\mathrm{HBOA}$ requires a relatively high computation time compared to other algorithms. However, in large nodes (50 nodes), the resulting computation time can compete with the TS algorithm. The high computation time of HBOA is caused by the large computation time TS algorithm that used to replace $10 \%$ of search agents BOA. Furthermore, the swap and flip procedure require repetition in each iteration, requiring a large computation time. In addition, this study used the sensory modality of 0.01 and power exponent of 0.1 from BOA parameters. In future investigations, it may be possible to use different sensory modality parameters and power exponent to test the quality of the solution (total distribution cost and computation time).

\section{Conclusion}

This study discussed the green vehicle routing problem (GVRP). The main objective of this research was to develop HBOA to minimize the distribution costs on G-VRP. This research successfully developed HBOA to solve G-VRP. The HBOA is proposed based on the BOA, which is improved with TS and local search procedures such as swap and flip. The experimental results show that the increase in population parameters and the HBOA iteration can minimize the total distribution costs. To test the algorithm performance, this algorithm was compared with several procedures. The experimental results proved that the HBOA produced a minimum total distribution cost than other algorithms. Therefore, the proposed algorithm is more competitive than the comparison algorithm. In the computation time, the results showed that the number of nodes significantly affects the computational time in HBOA. However, the proposed algorithm provides a relatively higher computation time compared to several other algorithms. Therefore, further research needs to be done to reduce the computation time so that the algorithm may become more efficient. Moreover, sensory modality and power exponent parameters need to be tested at various values. Future research should also aim at developing algorithms and problems with dynamic vehicle speeds, multidepot (distribution centers), and perishable products.

\section{Data Availability}

All data generated or analyzed during this study are included in this article.

\section{Conflicts of Interest}

The authors declare that they have no conflicts of interest.

\section{Acknowledgments}

The authors would like to thank the Directorate of the Research University of Muhammadiyah Malang for supporting the research and would also like to extend their gratitude to the Department of Industrial Engineering Optimization Laboratory for providing facilities.

\section{References}

[1] G. Poonthalir and R. Nadarajan, "Green vehicle routing problem with queues," Expert Systems with Applications, vol. 138, Article ID 112823, 2019.

[2] N. Rezaei, S. Ebrahimnejad, A. Moosavi, and A. Nikfarjam, "A green vehicle routing problem with time windows considering the heterogeneous fleet of vehicles: two metaheuristic algorithms," European Journal of Industrial Engineering, vol. 13, pp. 507-535, 2019.

[3] M. Syafrizal, B. Sugiarto, S. S. Moersidik, J. Fortin, N. Hamani, and E. Bretagne, "Dynamic vehicle emissions reduction with technical and behavioral approach," International Journal of Technology, vol. 7, no. 5, pp. 871-880, 2016.

[4] M. F. Ibrahim, M. M. Putri, and D. M. Utama, "A literature review on reducing carbon emission from supply chain system: drivers, barriers, performance indicators, and practices," IOP Conference Series: Materials Science and Engineering, vol. 722, Article ID 012034, 2020.

[5] J. Li and J. Zhang, "Study on the effect of carbon emission trading mechanism on logistics distribution routing decisions," System Engineering Theory and Practice, vol. 34, pp. 1779-1787, 2014.

[6] Y. Yu, S. Wang, J. Wang, and M. Huang, "A branch-and-price algorithm for the heterogeneous fleet green vehicle routing problem with time windows," Transportation Research Part B: Methodological, vol. 122, pp. 511-527, 2019.

[7] J. Zhang, Y. Zhao, W. Xue, and J. Li, "Vehicle routing problem with fuel consumption and carbon emission," International Journal of Production Economics, vol. 170, pp. 234-242, 2015.

[8] Y. Zhou, J.-B. Sheu, and J. Wang, "Robustness assessment of urban road network with consideration of multiple hazard events," Risk Analysis, vol. 37, no. 8, pp. 1477-1494, 2017.

[9] Ç. Koç, M. Erbaş, and E. Özceylan, "The impact of routing on $\mathrm{CO}_{2}$ emissions at a retail grocery store chain: a GIS-based solution approach," in Lean and Green Supply Chain Management, pp. 143-160, Springer, Berlin, Germany, 2019.

[10] L. Wang and J. Lu, "A memetic algorithm with competition for the capacitated green vehicle routing problem," IEEE/CAA Journal of Automatica Sinica, vol. 6, no. 2, pp. 516-526, 2019.

[11] G. Y. Qin, F. M. Tao, and L. X. Li, "A green vehicle routing optimization model with adaptive vehicle speed under soft time window," in Proceedings of the 2019 IEEE International Conference on Industrial Engineering and Engineering Management (IEEM), pp. 1-5, Macao, China, December 2019.

[12] G. Kim, Y.-S. Ong, C. K. Heng, P. S. Tan, and N. A. Zhang, "City vehicle routing problem (city VRP): a review," IEEE Transactions on Intelligent Transportation Systems, vol. 16, no. 4, pp. 1654-1666, 2015.

[13] D. M. Utama, S. K. Dewi, A. Wahid, and I. Santoso, "The vehicle routing problem for perishable goods: a systematic review," Cogent Engineering, vol. 7, Article ID 1816148, 2020.

[14] I. Masudin, R. F. Sa'diyah, R. F. Sa'diyah, D. M. Utama, D. P. Restuputri, and F. Jie, "Capacitated vehicle routing problems: nearest neighbour vs. Tabu search," International Journal of Computer Theory and Engineering, vol. 11, no. 4, pp. 76-79, 2019.

[15] F. A. Gunadi, D. Lesmono, and K. Aritonang, "A routing model for hybrid hub-and-spoke with time windows," Jurnal Teknik Industri, vol. 21, no. 1, pp. 22-33, 2020. 
[16] G. B. Dantzig and J. H. Ramser, "The truck dispatching problem," Management Science, vol. 6, no. 1, pp. 80-91, 1959.

[17] J. Mandziuk, "New shades of the vehicle routing problem: emerging problem formulations and computational intelligence solution methods," IEEE Transactions on Emerging Topics in Computational Intelligence, vol. 3, no. 3, pp. 230244, 2019.

[18] D. N. Nenchev, A. Miyata, S. Miyahara, and T. Hamano, "The VRP generalized inverse and its application in DCM/VRPbased walking control," IEEE Robotics and Automation Letters, vol. 4, no. 4, pp. 4595-4602, 2019.

[19] Y. Su and Q.-M. Fan, "The green vehicle routing problem from a smart logistics perspective," IEEE Access, vol. 8, pp. 839-846, 2020.

[20] G. Qin, F. Tao, and L. Li, "A vehicle routing optimization problem for cold chain logistics considering customer satisfaction and carbon emissions," International Journal of Environmental Research and Public Health, vol. 16, no. 4, p. 576, 2019.

[21] J. Oesterle and T. Bauernhansl, "Exact method for the vehicle routing problem with mixed linehaul and backhaul customers, heterogeneous fleet, time window and manufacturing capacity," Procedia CIRP, vol. 41, pp. 573-578, 2016.

[22] J. Dethloff, "Vehicle routing and reverse logistics: the vehicle routing problem with simultaneous delivery and pick-up," $O R$ Spektrum, vol. 23, no. 1, pp. 79-96, 2001.

[23] K. Braekers, K. Ramaekers, and I. Van Nieuwenhuyse, "The vehicle routing problem: state of the art classification and review," Computers \& Industrial Engineering, vol. 99, pp. 300-313, 2016.

[24] F. E. Zulvia, R. J. Kuo, and D. Y. Nugroho, "A many-objective gradient evolution algorithm for solving a green vehicle routing problem with time windows and time dependency for perishable products," Journal of Cleaner Production, vol. 242, Article ID 118428, 2020.

[25] P. L. N. U. Cooray and T. D. Rupasinghe, "Machine learningbased parameter tuned genetic algorithm for energy minimizing vehicle routing problem," Journal of Industrial Engineering, vol. 2017, Article ID 3019523, 13 pages, 2017.

[26] G. Poonthalir and R. Nadarajan, "A fuel efficient green vehicle routing problem with varying speed constraint (F-GVRP)," Expert Systems with Applications, vol. 100, pp. 131-144, 2018.

[27] N. Norouzi, M. Sadegh-Amalnick, and R. Tavakkoli-Moghaddam, "Modified particle swarm optimization in a time-dependent vehicle routing problem: minimizing fuel consumption," Optimization Letters, vol. 11, no. 1, pp. 121-134, 2017.

[28] S. Zhang, W. Zhang, Y. Gajpal, and S. Appadoo, "Ant colony algorithm for routing alternate fuel vehicles in multi-depot vehicle routing problem," in Decision Science in Action, pp. 251-260, Springer, Berlin, Germany, 2019.

[29] Y. Kuo, "Using simulated annealing to minimize fuel consumption for the time-dependent vehicle routing problem," Computers \& Industrial Engineering, vol. 59, no. 1, pp. 157$165,2010$.

[30] N. M. E. Normasari, V. F. Yu, C. Bachtiyar, and Sukoyo, "A simulated annealing heuristic for the capacitated green vehicle routing problem," Mathematical Problems in Engineering, vol. 2019, Article ID 2358258, 18 pages, 2019.

[31] R. Wang, J. Zhou, X. Yi, and A. A. Pantelous, "Solving the green-fuzzy vehicle routing problem using a revised hybrid intelligent algorithm," Journal of Ambient Intelligence and Humanized Computing, vol. 10, no. 1, pp. 321-332, 2018.

[32] J. Andelmin and E. Bartolini, "A multi-start local search heuristic for the green vehicle routing problem based on a multigraph reformulation," Computers \& Operations Research, vol. 109, pp. 43-63, 2019.

[33] G. Macrina, G. Laporte, F. Guerriero, and L. Di Puglia Pugliese, "An energy-efficient green-vehicle routing problem with mixed vehicle fleet, partial battery recharging and time windows," European Journal of Operational Research, vol. 276, no. 3, pp. 971-982, 2019.

[34] E. E. Adiba, E. A. Aahmed, and B. Youssef, "The green capacitated vehicle routing problem: optimizing of emissions of greenhouse gas," in Proceedings of the 2014 International Conference on Logistics Operations Management, pp. 161-167, Rabat, Morocco, June 2014.

[35] A. Sruthi, S. P. Anbuudayasankar, G. Jeyakumar, and S. C. Management, "Energy-efficient green vehicle routing problem," International Journal of Information Systems and Supply Chain Management, vol. 12, no. 4, pp. 27-41, 2019.

[36] O. Jabali, T. Van Woensel, A. G. De Kok, and O. Management, "Analysis of travel times and $\mathrm{CO}_{2}$ emissions in time-dependent vehicle routing," Production and Operations Management, vol. 21, no. 6, pp. 1060-1074, 2012.

[37] P. Kirci, "On the performance of Tabu search algorithm for the vehicle routing problem with time windows," in Proceedings of the 2016 IEEE 4th International Conference on Future Internet of Things and Cloud Workshops (FiCloudW), pp. 351-354, Vienna, Austria, August 2016.

[38] K. Udin, R. Gui, and A. Rahmawan, "Green vehicle routing problem with heterogeneous fleet and time windows," in Proceedings of the 6th International Conference on Software and Computer Applications, pp. 223-227, Bangkok, Thailand, February 2017.

[39] H. Li, J. Yuan, T. Lv, and X. Chang, "The two-echelon timeconstrained vehicle routing problem in linehaul-delivery systems considering carbon dioxide emissions," Transportation Research Part D: Transport and Environment, vol. 49, pp. 231-245, 2016.

[40] Y. Xiao and A. Konak, "A genetic algorithm with exact dynamic programming for the green vehicle routing \& scheduling problem," Journal of Cleaner Production, vol. 167, pp. 1450-1463, 2017.

[41] S. Kunnapapdeelert and R. Klinsrisuk, "Determination of green vehicle routing problem via differential evolution," International Journal of Logistics Systems and Management, vol. 34, no. 3, pp. 395-410, 2019.

[42] J. C. Molina, I. Eguia, and J. Racero, "Reducing pollutant emissions in a waste collection vehicle routing problem using a variable neighborhood tabu search algorithm: a case study," TOP, vol. 27, no. 2, pp. 253-287, 2019.

[43] Y. Li, M. K. Lim, and M.-L. Tseng, "A green vehicle routing model based on modified particle swarm optimization for cold chain logistics," Industrial Management \& Data Systems, vol. 119, no. 3, pp. 473-494, 2019.

[44] S. Wang, F. Tao, and Y. Shi, "Optimization of location-routing problem for cold chain logistics considering carbon footprint," International Journal of Environmental Research and Public Health, vol. 15, no. 1, p. 86, 2018.

[45] L. Shen, F. Tao, and S. Wang, "Multi-depot open vehicle routing problem with time windows based on carbon trading," International Journal of Environmental Research and Public Health, vol. 15, no. 9, p. 2025, 2018.

[46] Y. Li, H. Soleimani, and M. Zohal, “An improved ant colony optimization algorithm for the multi-depot green vehicle routing problem with multiple objectives," Journal of Cleaner Production, vol. 227, pp. 1161-1172, 2019. 
[47] K. Karagul, Y. Sahin, E. Aydemir, and A. Oral, "A simulated annealing algorithm based solution method for a green vehicle routing problem with fuel consumption," in Lean and Green Supply Chain Management: Optimization Models and Algorithms, T. Paksoy, G.-W. Weber, and S. Huber, Eds., pp. 161-187, Springer International Publishing, Cham, Switzerland, 2019.

[48] S. Arora and S. Singh, "Butterfly optimization algorithm: a novel approach for global optimization," Soft Computing, vol. 23, pp. 715-734, 2019.

[49] L. Wen and Y. Cao, "A hybrid intelligent predicting model for exploring household $\mathrm{CO}_{2}$ emissions mitigation strategies derived from butterfly optimization algorithm," Science of the Total Environment, vol. 727, Article ID 138572, 2020.

[50] T. K. Sharma, A. Kumar Sahoo, and P. Goyal, "Bidirectional butterfly optimization algorithm and engineering applications," Materials Today: Proceedings, 2020.

[51] B. S. Yıldız, A. R. Yıldı, E. İ. Albak, H. Abderazek, S. M. Sait, and S. Bureerat, "Butterfly optimization algorithm for optimum shape design of automobile suspension components," Materials Testing, vol. 62, pp. 365-370, 2020.

[52] D. M. Utama, "An effective hybrid sine cosine algorithm to minimize carbon emission on flow-shop scheduling sequence dependent setup," Jurnal Teknik Industri, vol. 20, pp. 62-72, 2019.

[53] D. M. Utama, D. S. Widodo, M. F. Ibrahim, and S. K. Dewi, "An effective hybrid ant lion algorithm to minimize mean tardiness on permutation flow shop scheduling problem," International Journal of Advances in Intelligent Informatics, vol. 6, no. 1, pp. 23-35, 2020.

[54] D. M. Utama, D. S. Widodo, M. F. Ibrahim, K. Hidayat, T. Baroto, and A. Yurifah, "The hybrid whale optimization algorithm: a new metaheuristic algorithm for energy-efficient on flow shop with dependent sequence setup," Journal of Physics: Conference Series, vol. 1569, Article ID 022094, 2020.

[55] D. S. Widodo and D. M. Utama, "The hybrid ant lion optimization flow shop scheduling problem for minimizing completion time," Journal of Physics: Conference Series, vol. 1569, Article ID 022097, 2020.

[56] T. J. Gaskell, "Bases for vehicle fleet scheduling," Journal of the Operational Research Society, vol. 18, pp. 281-295, 1967.

[57] N. Christofides and S. Eilon, "An algorithm for the vehicledispatching problem," Journal of the Operational Research Society, vol. 20, pp. 309-318, 1969.

[58] C. Franco, E. R. López-Santana, and J. C. Figueroa-García, "Solving the interval green inventory routing problem using optimization and genetic algorithms," in Proceedings of the Workshop on Engineering Applications, pp. 556-564, Cartagena, Colombia, September 2017. 